\title{
Charge Trapping in Monolayer and Multilayer Epitaxial Graphene
}

\section{Chieh-I Liu, ${ }^{1}$ Pengjie Wang, ${ }^{2}$ Jian Mi, ${ }^{2}$ Hsin-Yen Lee, ${ }^{3}$ Chi Zhang, ${ }^{2}$ Xi Lin, ${ }^{2}$ Chiashain Chuang, ${ }^{4,5}$ Nobuyuki Aoki, ${ }^{4}$ Randolph E. Elmquist, ${ }^{5}$ and Chi-Te Liang ${ }^{1,3}$}

\author{
${ }^{1}$ Graduate Institute of Applied Physics, National Taiwan University, Taipei 106, Taiwan \\ ${ }^{2}$ International Center for Quantum Materials, Peking University, Beijing 100871, China \\ ${ }^{3}$ Department of Physics, National Taiwan University, Taipei 106, Taiwan \\ ${ }^{4}$ Graduate School of Advanced Integration Science, Chiba University, Chiba 263-8522, Japan \\ ${ }^{5}$ National Institute of Standards and Technology (NIST), Gaithersburg, MD 20899, USA
}

Correspondence should be addressed to Hsin-Yen Lee; hyli.ai@gmail.com, Chiashain Chuang; chiashain@gmail.com, and Chi-Te Liang; ctliang@phys.ntu.edu.tw

Received 11 December 2015; Accepted 10 March 2016

Academic Editor: Ungyu Paik

Copyright ( 2016 Chieh-I Liu et al. This is an open access article distributed under the Creative Commons Attribution License, which permits unrestricted use, distribution, and reproduction in any medium, provided the original work is properly cited.

\begin{abstract}
We have studied the carrier densities $n$ of multilayer and monolayer epitaxial graphene devices over a wide range of temperatures $T$. It is found that, in the high temperature regime (typically $T \geq 200 \mathrm{~K}$ ), $\ln (n)$ shows a linear dependence of $1 / T$, showing activated behavior. Such results yield activation energies $\Delta E$ for charge trapping in epitaxial graphene ranging from $196 \mathrm{meV}$ to $34 \mathrm{meV}$. We find that $\Delta E$ decreases with increasing mobility. Vacuum annealing experiments suggest that both adsorbates on EG and the $\mathrm{SiC} /$ graphene interface play a role in charge trapping in EG devices.
\end{abstract}

Graphene, a layer of carbon atoms bonded in a hexagonal lattice, has attracted a great deal of worldwide interest [1, 2] because of its fundamental importance as well as its great potential applications. Although graphene prepared by the mechanical exfoliation method $[3,4]$ has the highest sample quality which allows one to study fascinating physical phenomena, its small size may limit graphene's realworld applications. In contrast, the sizes of chemical vapor deposition-grown graphene $[5,6]$ and epitaxial graphene (EG) grown on $\mathrm{SiC}[7-12]$ can be large and thus they are highly desirable for wafer-scale device applications. In particular, EG can find prospects in high-frequency transistors [13] and thus is a great candidate for integration of graphenebased $\mathrm{GHz}$ devices with existing Si technology. In order to fully realize EG's potential, it is highly desirable to obtain a thorough understanding of the electronic properties of epitaxial graphene, for example, how the carrier density of an EG device varies with measurement temperature. Such important information can shed light on charge trapping in $\mathrm{EG}$ grown on $\mathrm{SiC}$ [11]. In this paper, we report carrier density (n) measurements of two multilayer and two monolayer graphene devices over a wide range of temperatures $(T)$. We find that, in the high temperature regime, $\ln (n)$ shows a linear dependence on $1 / T$. Such results yield activation energies for charge trapping ranging from $196 \mathrm{meV}$ to $34 \mathrm{meV}$ depending on the quality of the EG devices. By low-temperature vacuum annealing to remove adsorbates on a monolayer EG device, we are able to vary the carrier density of the sample in a controlled manner without significantly modifying the nature of the SiC/graphene interface. We suggest that both the $\mathrm{SiC} /$ graphene interface and the adsorbates on EG devices must be considered in order to obtain a thorough understanding of charge trapping in EG devices. Our results further suggest that, by low-temperature vacuum annealing $(T \sim$ $400 \mathrm{~K}$ ), the carrier density of monolayer EG can be roughly constant, a great advantage for electronic devices operating over a wide range of temperatures.

Here we briefly describe fabrication of multilayer EG samples. SiC substrates were cleaned using a standard substrate cleaning procedure. The optically polished $\mathrm{Si}$-face 


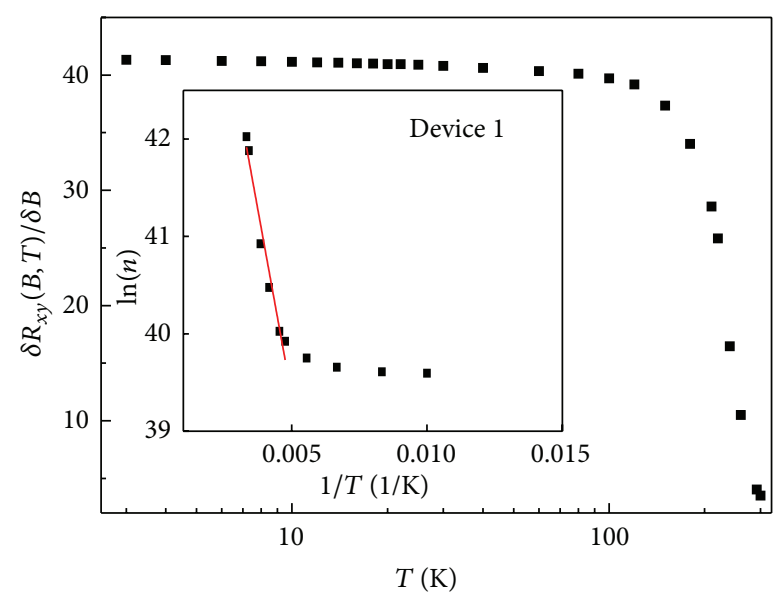

FIGURE 1: Hall slope as a function of temperature on a semilogarithmic scale (Device 1). The inset shows $\ln (n)$ as a function of $1 / T$. The linear fit yields the activation energy.

surfaces were then placed face to face with a polished graphite disk and arranged such that uniform Newton rings could be observed in fluorescent light [14]. These optically finished substrate surfaces, as compared to the ones that were treated by the chemical-mechanical processed (CMP) method, resulted in a higher rate of $\mathrm{SiC}$ decomposition and created multiple graphene layers. The epitaxial growth process was controlled by annealing in a sequence of temperature ramps and dwell stages in Ar gas at a pressure slightly higher than $1 \mathrm{~atm}$ using a commercial furnace. After dehydration and cleaning at $725^{\circ} \mathrm{C}$ for 16 hours in the furnace, the temperature was ramped to $1200^{\circ} \mathrm{C}$ for $30 \mathrm{~min}$ and then ramped at $100^{\circ} \mathrm{C} / \mathrm{min}$ for graphene growth at a temperature of $1850^{\circ} \mathrm{C}$ and $1950^{\circ} \mathrm{C}$ with a dwell time of $45 \mathrm{~min}$ and $30 \mathrm{~min}$ for Device 1 and Device 2, respectively. The temperatures were measured and controlled using molybdenum-sheathed type "C" thermocouples. After being carefully examined by the tapping-mode atomic force microscopy (AFM) and the transmission electron microscopy (TEM), Device 1 was estimated to have five layers, and Device 2 was estimated to have six layers. Optically polished substrates produce much thicker graphene for the same processing conditions compared to that grown on CMP surfaces.

Monolayer epitaxial graphene samples (Device 3 and Device 4) were grown on an optically polished $6 \mathrm{H}-\mathrm{SiC}(0001)$ wafer at $1850^{\circ} \mathrm{C}$ for 30 minutes under an $\mathrm{Ar}$ gas pressure at $98 \mathrm{kPa}$ using a controlled $\mathrm{Si}$ sublimation process [15]. The number of layers was confirmed by the AFM images. Specifically, we deposited a metal bilayer $(5 \mathrm{~nm}$ thick Pd and $10 \mathrm{~nm}$ thick $\mathrm{Au}$ ) directly on the as-grown EG after a dehydration baking at $T=115^{\circ} \mathrm{C}$ for 10 minutes so as to prevent the contamination from photoresist residues so as to produce low-carrier-density epitaxial graphene devices at low temperatures. We performed the standard optical lithography for a Hall-bar configuration and the protective metal was removed from the Hall bars using diluted aqua regia before

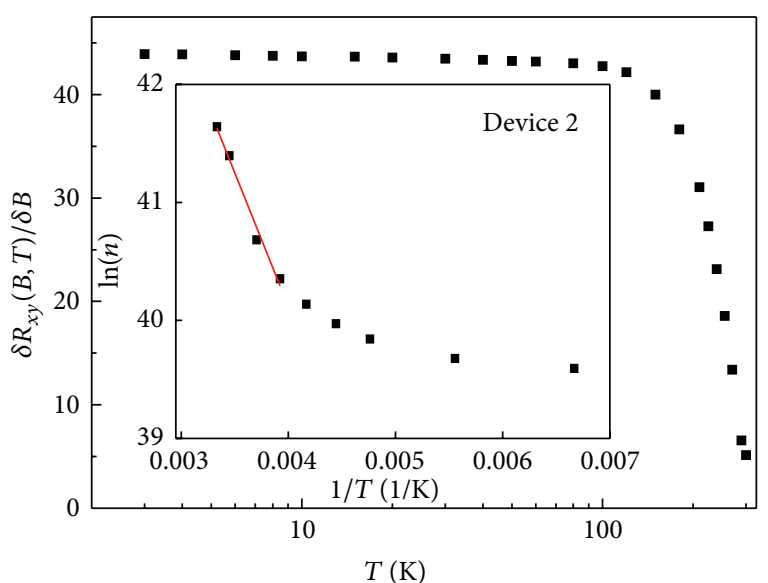

FIgURE 2: Hall slope as a function of temperature on a semilogarithmic scale (Device 2 ). The inset shows $\ln (n)$ as a function of $1 / T$. The linear fit yields the activation energy.

measuring the device [15]. Four-terminal magnetoresistivities for longitudinal $\rho_{x x}$ and Hall $R_{x y}$ were measured using standard AC lock-in techniques.

Figures 1 and 2 show the measured Hall slope $\delta R_{x y}(B, T) / \delta B$ of multilayer EG samples (Device 1 and Device 2) as a function of $T$, respectively. The change of the low-temperature Hall slope can be ascribed to electron-electron $(e-e)$ interactions [16]. However, at high temperatures, the observed change of the Hall slope is large ( $>10$-fold) and cannot be simply to $e-e$ interactions. Therefore this large change in the Hall slope at high $T$ must be related to the change of $n$ with increasing $T$. In order to further study this interesting effect, we plot the logarithmic scale of the measured carrier density $\ln (n)$ as a function of $1 / T$ (see the inset of Figures 1 and 2). Interestingly, in the high temperature regime ( $T \geq 200 \mathrm{~K})$, the data can be fitted to the following equation:

$$
\ln (n)=-\left(\frac{\Delta E}{k_{\mathrm{B}}}\right) \frac{1}{T}+C,
$$

where $\Delta E, k_{\mathrm{B}}$, and $C$ are the activation energy, the Boltzmann constant, and the fitting constant, respectively. The measured $\Delta E$ for Device 1 is $(132 \pm 12) \mathrm{meV}$ which is the energy for electrons trapping. Similar results are obtained on Device 2 with a slightly higher $\Delta E=(196 \pm 17) \mathrm{meV}$. Our results are in qualitative agreement with those obtained on front-gated monolayer graphene ( 70 meV) [10].

It is natural to study the charge trapping effect in monolayer EG. To this end, we plot $\ln (n)$ as a function of $1 / T$ for Device 3 and Device 4 in Figure 3 and the inset in Figure 3, respectively. The measured $\Delta E$ is $(50 \pm 1) \mathrm{meV}$ and $(34 \pm$ 4) $\mathrm{meV}$ for Device 3 and Device 4 . Such values are smaller than those obtained in our more disordered multilayer EG devices. Since the mobilities, which can be calculated from Hall slope and the longitudinal resistivity at zero magnetic field, of our monolayer graphene $\left(>30000 \mathrm{~cm}^{2} / \mathrm{V} / \mathrm{s}\right.$ at $T=$ $3 \mathrm{~K})$ are much higher than those of multilayer graphene $\left(\sim 1000 \mathrm{~cm}^{2} / \mathrm{V} / \mathrm{s}\right.$ at $\left.T=3 \mathrm{~K}\right)$ due to homogenous film with less 


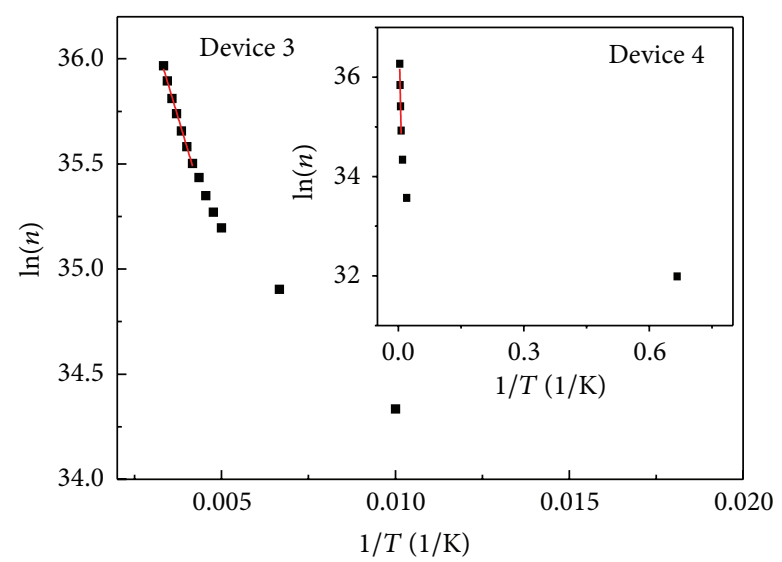

FIgURE $3: \ln (n)$ as a function of $1 / T$. The linear fit yields the activation energy (Device 3 ). The inset shows $\ln (n)$ as a function of $1 / T$. The linear fit yields the activation energy (Device 4 ).

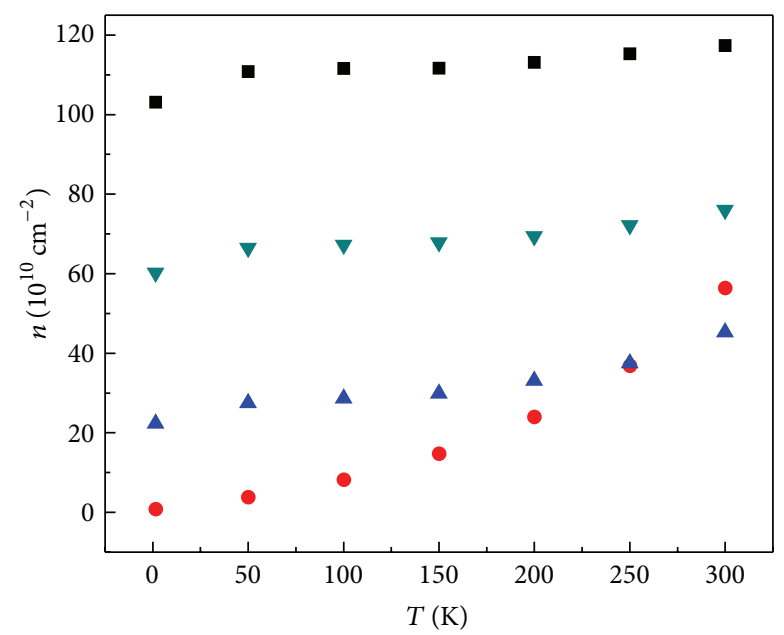

FIGURE 4: $n(T)$ under different vacuum annealing conditions. From bottom to top: as prepared, annealing at $T=383 \mathrm{~K}$ for 5 minutes, annealing at $T=393 \mathrm{~K}$ for 5 minutes, and annealing at $T=423 \mathrm{~K}$ for 5 minutes, respectively.

disordered conditions in monolayer graphene, our results suggest that, with increasing mobility, there is less charge trapping. The carrier mobility in the monolayer was greater than that in the multilayer due to the linear dispersion relation [17].

By vacuum annealing to remove adsorbates on monolayer EG, we are able to controllably vary the low-temperature carrier density of our devices. Figure 4 shows $n(T)$ under different low- $T$ annealing conditions. For $n \geq 2.2 \times 10^{11} \mathrm{~cm}^{-2}$, we can see that $n$ only increases slightly when increasing the measurement temperature. In these cases, the change in $n$ is too small and fitting the data to (1) yields $\Delta E$ smaller than the thermal energy at $300 \mathrm{~K}$ which is unphysical. We note that in top-gated monolayer EG charge trapping occurs at the $\mathrm{SiC}$ substrate since the surfaces of the devices are protected by a dielectric layer [11]. In our case, since the annealing temperature is low $(T \leq 423 \mathrm{~K})$ and is not expected to vary

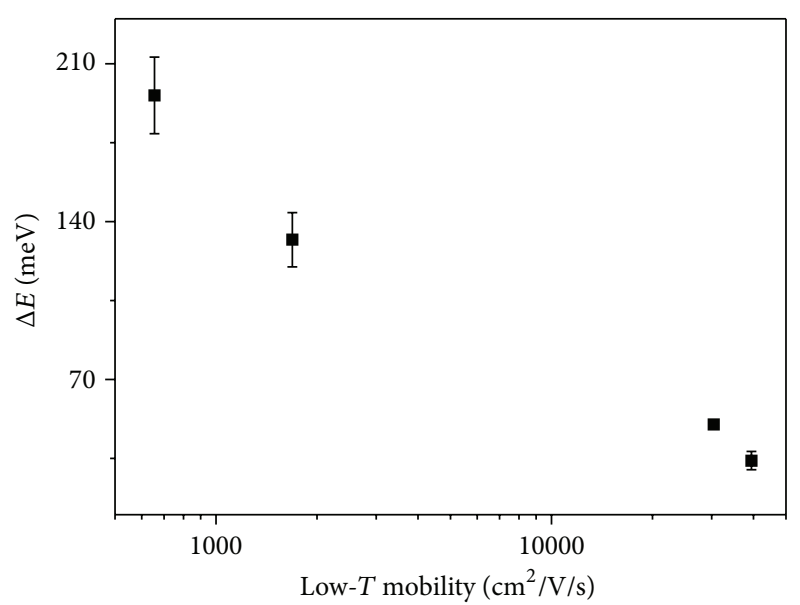

FIgURE 5: $\Delta E$ as function of low- $T$ mobility on a semilogarithmic scale. From left to right: Device 2, Device 1, Device 3, and Device 4.

the nature of the $\mathrm{SiC} /$ graphene interface [18], our results show that the adsorbates on EG can significantly vary the binding energy for charge trapping. We thus suggest that one must consider charge trapping [19-21] by both SiC/graphene interface and adsorbates on graphene in open-faced EG.

Finally Figure 5 shows the measured $\Delta E$ as a function of low-temperature mobility $(\mu)$. We can immediately see that $\Delta E$ decreases with increasing $\mu$. Our results demonstrate that the binding energy for charge trapping decreases with sample quality. Charge trapping, which could be detrimental to device operation over a wide range of $T$, should be minimized by improving the sample quality including the $\mathrm{SiC} /$ graphene interface, the graphene surfaces, and the sample mobility.

In conclusion, we have reported carrier density measurements of multilayer and monolayer epitaxial graphene devices over a wide range of temperatures. It is found that the activation energies corresponding to charge trapping decrease with increasing sample mobility. Vacuum annealing experiments show that both adsorbates on EG's surface and the $\mathrm{SiC} /$ graphene interface play an important role in determining the carrier density dependence on $T$.

\section{Competing Interests}

The authors declare that they have no competing interests.

\section{Acknowledgments}

Hsin-Yen Lee was supported by National Taiwan University (Grant no. 103R4000). Chi Zhang was supported by the 973 Program: NBRP of China (2013CB921903 and 2013CB920904). Xi Lin was supported by the NSFC (Grants nos. 11274020 and 11322435). Chi-Te Liang acknowledges support from National Taiwan University (Grants nos. 104R75522 and 105R890932). As an International Research Fellow of the Japan Society for the Promotion of Science (JSPS), Chiashain Chuang acknowledges the JSPS Postdoctoral Fellowship. The authors would like to thank Yi-Ting Wang and Yanfei Yang for experimental help. 


\section{References}

[1] K. S. Novoselov, A. K. Geim, S. V. Morozov et al., "Electric field in atomically thin carbon films," Science, vol. 306, no. 5696, pp. 666-669, 2004.

[2] V. Dhand, K. Y. Rhee, H. Ju Kim, and D. Ho Jung, "A comprehensive review of graphene nanocomposites: research status and trends," Journal of Nanomaterials, vol. 2013, Article ID 763953, 14 pages, 2013.

[3] Y. Zhang, Y.-W. Tan, H. L. Stormer, and P. Kim, "Experimental observation of the quantum Hall effect and Berry's phase in graphene," Nature, vol. 438, no. 7065, pp. 201-204, 2005.

[4] K. S. Novoselov, A. K. Geim, S. V. Morozov et al., "Twodimensional gas of massless Dirac fermions in graphene," Nature, vol. 438, no. 7065, pp. 197-200, 2005.

[5] X. Li, W. Cai, J. An et al., "Large-area synthesis of high-quality and uniform graphene films on copper foils," Science, vol. 324, no. 5932, pp. 1312-1314, 2009.

[6] Y.-P. Hsieh, Y.-W. Wang, C.-C. Ting, H.-C. Wang, K.-Y. Chen, and C.-C. Yang, "Effect of catalyst morphology on the quality of CVD grown graphene," Journal of Nanomaterials, vol. 2013, Article ID 393724, 6 pages, 2013.

[7] C. Berger, Z. Song, T. Li et al., "Ultrathin epitaxial graphite: $2 \mathrm{D}$ electron gas properties and a route toward graphene-based nanoelectronics," Journal of Physical Chemistry B, vol. 108, no. 52, pp. 19912-19916, 2004.

[8] T. Rana, M. V.S. Chandrashekhar, K. Daniels, and T. Sudarshan, "Epitaxial growth of graphene on $\mathrm{SiC}$ by $\mathrm{Si}$ selective etching using $\mathrm{SiF}_{4}$ in an inert ambient," Japanese Journal of Applied Physics, vol. 54, no. 3, Article ID 030304, 2015.

[9] K. V. Emtsev, A. Bostwick, K. Horn et al., "Towards wafersize graphene layers by atmospheric pressure graphitization of silicon carbide," Nature Materials, vol. 8, no. 3, pp. 203-207, 2009.

[10] H. Hibino, H. Kageshima, and M. Nagase, "Epitaxial few-layer graphene: towards single crystal growth," Journal of Physics D: Applied Physics, vol. 43, no. 37, Article ID 374005, 2010.

[11] D. B. Farmer, V. Perebeinos, Y.-M. Lin, C. Dimitrakopoulos, and P. Avouris, "Charge trapping and scattering in epitaxial graphene," Physical Review B-Condensed Matter and Materials Physics, vol. 84, no. 20, Article ID 205417, 2011.

[12] M. Nagase, H. Hibino, H. Kageshima, and H. Yamaguchi, "Graphene-based nano-electro-mechanical switch with high on/off ratio," Applied Physics Express, vol. 6, no. 5, Article ID 055101, 2013.

[13] Y.-M. Lin, C. Dimitrakopoulos, K. A. Jenkins et al., "100-GHz transistors from wafer-scale epitaxial graphene," Science, vol. 327, no. 5966, p. 662, 2010.

[14] M. A. Real, E. A. Lass, F.-H. Liu et al., "Graphene epitaxial growth on $\mathrm{SiC}(0001)$ for resistance standards," IEEE Transactions on Instrumentation and Measurement, vol. 62, no. 6, pp. 1454-1460, 2013.

[15] Y. Yang, L.-I. Huang, Y. Fukuyama et al., "Low carrier density epitaxial graphene devices on SiC", Small, vol. 11, no. 1, pp. 9095, 2015.

[16] M. Y. Simmons, A. R. Hamilton, M. Pepper, E. H. Linfield, P. D. Rose, and D. A. Ritchie, "Weak localization, hole-hole interactions, and the "Metal"-insulator transition in two dimensions," Physical Review Letters, vol. 84, no. 11, pp. 2489-2492, 2000.

[17] K. Nagashio, T. Nishimura, K. Kita, and A. Toriumi, "Mobility variations in mono- and multi-layer graphene films," Applied Physics Express, vol. 2, no. 2, Article ID 025003, 2009.
[18] C. Riedl, C. Coletti, and U. Starke, "Structural and electronic properties of epitaxial graphene on $\mathrm{SiC}(0001)$ : a review of growth, characterization, transfer doping and hydrogen intercalation," Journal of Physics D: Applied Physics, vol. 43, no. 37, Article ID 374009, 2010.

[19] D. R. Hang, C.-T. Liang, J.-R. Juang et al., "Electrically detected and microwave-modulated Shubnikov-de Haas oscillations in an $\mathrm{Al}_{0.4} \mathrm{Ga}_{0.6} \mathrm{~N} / \mathrm{GaN}$ heterostructure," Journal of Applied Physics, vol. 93, no. 4, pp. 2055-2058, 2003.

[20] J. R. Juang, T.-Y. Huang, T.-M. Chen et al., "Transport in a gated $\mathrm{Al}_{0.18} \mathrm{Ga}_{0.82} \mathrm{~N} / \mathrm{GaN}$ electron system," Journal of Applied Physics, vol. 94, no. 5, pp. 3181-3184, 2003.

[21] J. H. Chen, J. Y. Lin, J. K. Tsai et al., "Experimental evidence for Drude-Boltzmann-like transport in a two-dimensional electron gas in an AlGaN/GaN heterostructure," Journal of the Korean Physical Society, vol. 48, no. 6, pp. 1539-1543, 2006. 

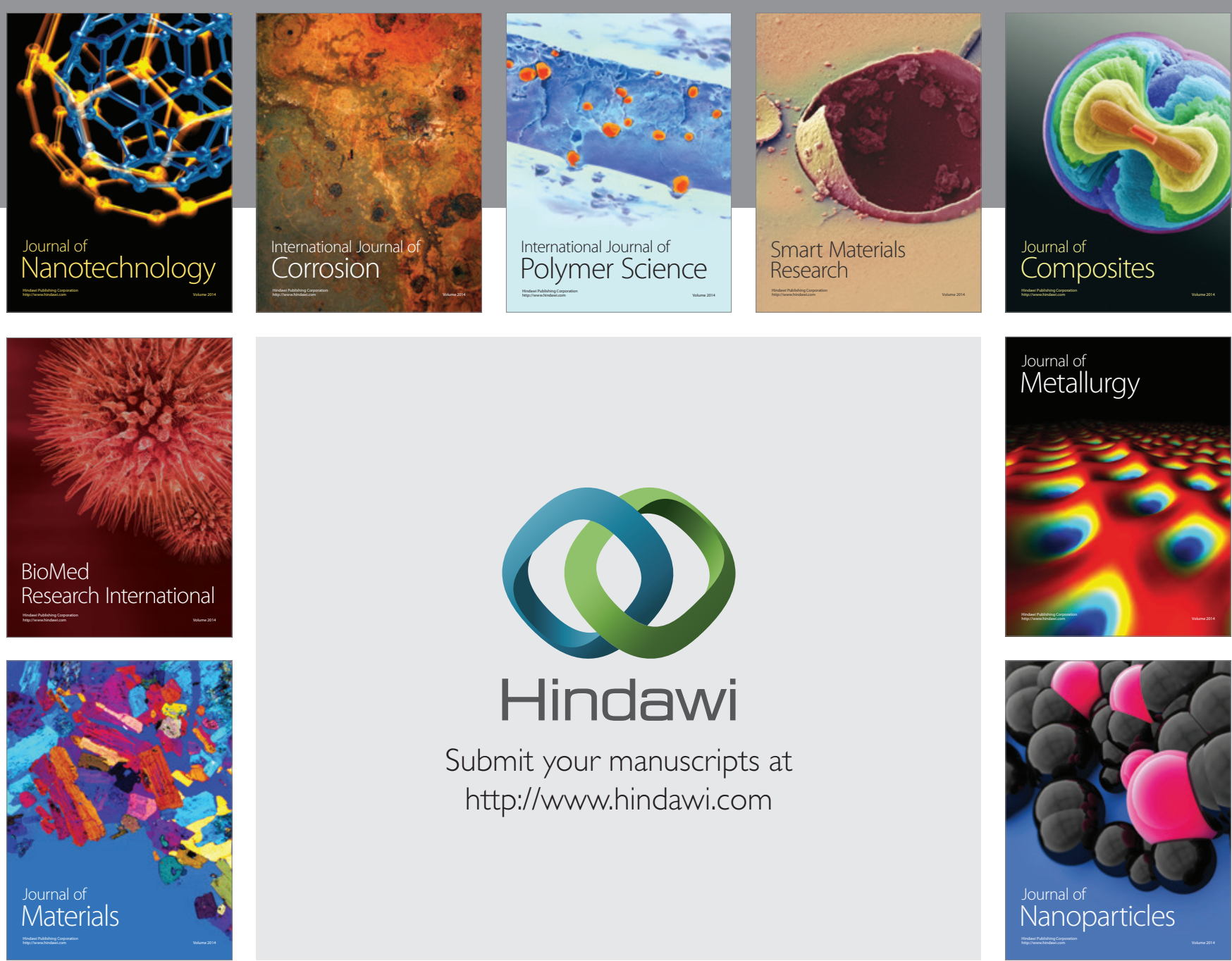

\section{Hindawi}

Submit your manuscripts at

http://www.hindawi.com

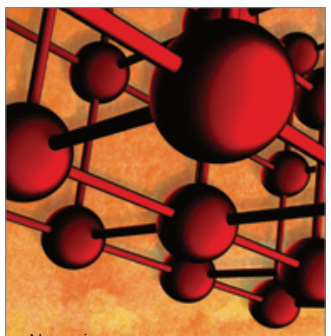

Materials Science and Engineering
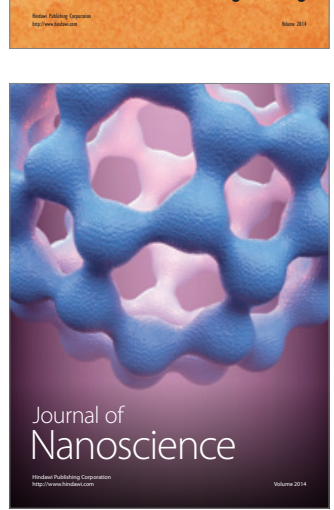
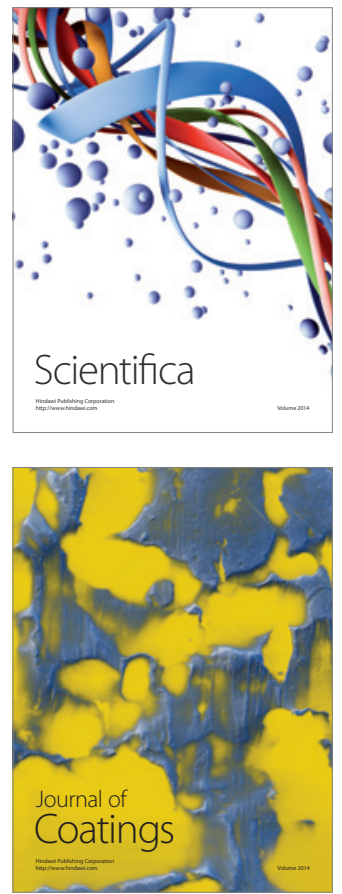
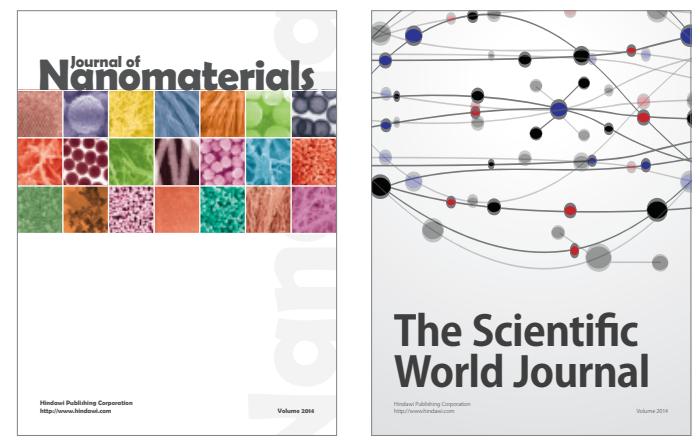

The Scientific World Journal
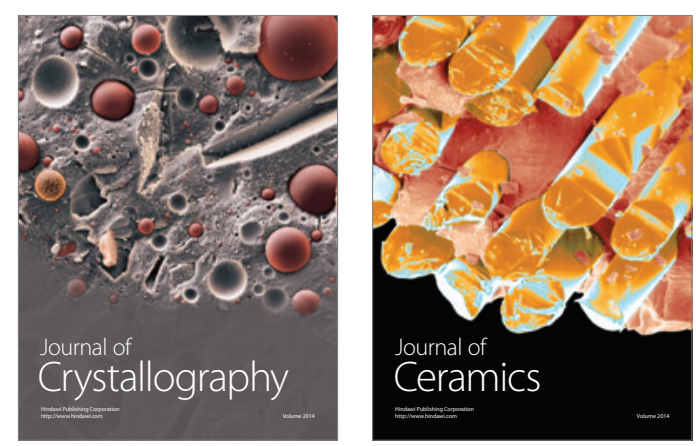
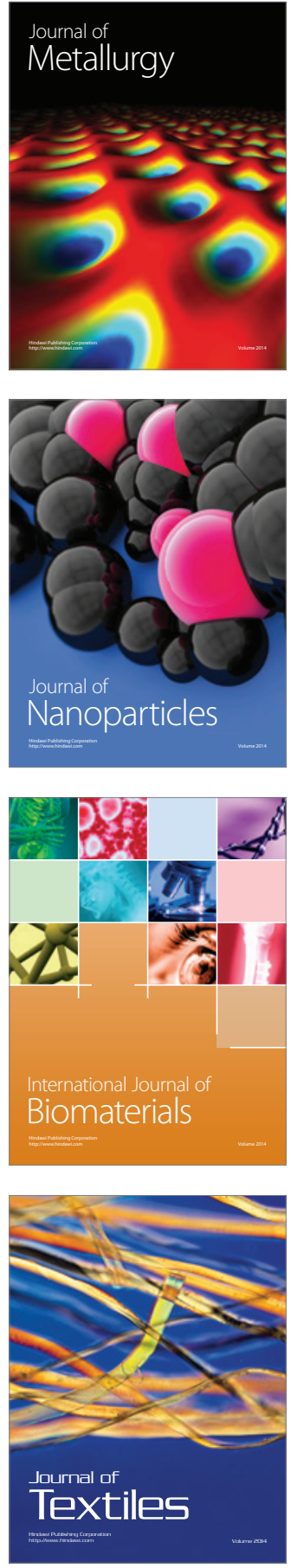